PROCEEDINGS OF THE

AMERICAN MATHEMATICAL SOCIETY

Volume 135, Number 12, December 2007, Pages 3815-3821

S 0002-9939(07)09005-3

Article electronically published on March 21, 2007

\title{
A TROPICAL NULLSTELLENSATZ
}

\author{
EUGENII SHUSTIN AND ZUR IZHAKIAN
}

(Communicated by Bernd Ulrich)

\begin{abstract}
We suggest a version of Nullstellensatz over the tropical semiring, the real numbers equipped with operations of maximum and summation.
\end{abstract}

\section{INTRODUCTION}

Tropical mathematics takes place in the tropical semiring, the real numbers equipped with the operations of maximum and summation, corresponding to the classical operations of addition and multiplication, respectively (see in [7, 17 the representation of the tropical semiring as a limit of the semiring of non-negative real numbers $\left.\left(\mathbb{R}_{+},+, \cdot\right)\right)$. Sometimes the tropical semiring is extended by $-\infty$, the neutral element for the maximum operation, but we shall not need it. We write the tropical operations in quotes, i.e.,

$$
" a+b "=\max \{a, b\}, \quad " a b "=a+b .
$$

A rapid development of tropical mathematics over the last years, especially of tropical algebraic geometry, has led to spectacular applications in classical algebraic geometry (see, for example, [2, 3, 4, 15, 8, 9, 10, 11, 13, 14, 15, 16, 17]). Tropical objects unexpectedly reveal much similarity with classical objects, which is based on the theory of large complex limits, logarithmic limits, non-Archimedean valuations, toric geometry, etc. Here we suggest the tropical analogue of Nullstellensatz (in a bit different context this problem was stated in [1, Question 16).

Similar to the classical case, we understand Nullstellensatz as a criterion for a polynomial to belong to the radical of an ideal. Let us give the necessary definitions (cf. [3, 11, 13, 14, 15, 17]). A tropical polynomial in $n$ variables is a function $F: \mathbb{R}^{n} \rightarrow \mathbb{R}$ given by

$$
F(x)=" \sum_{\omega \in \Omega} c_{\omega} x^{\omega} "=\max _{\omega \in \Omega}\left(\langle x, \omega\rangle+c_{\omega}\right),
$$

Received by the editors September 12, 2005 and, in revised form, July 29, 2006 and October $13,2006$.

2000 Mathematics Subject Classification. Primary 12K10, 13B25; Secondary 51M20.

Key words and phrases. Max-plus algebra, convex piecewise linear functions, polynomial ideals, Nullstellensatz.

The first author was supported by a grant from the High Council for Scientific and Technological Cooperation between France and Israel and by the grant no. 465/04 from the Israel Science Foundation.

The second author was supported by a grant from the High Council for Scientific and Technological Cooperation between France and Israel.

(C)2007 American Mathematical Society 
where $\Omega$ is a non-empty finite set of points in $\mathbb{Z}^{n}$ with non-negative coordinates, $\langle *, *\rangle$ denotes the scalar product, and $c_{\omega} \in \mathbb{R}, \omega \in \Omega$. This is a convex piecewise linear function. With a tropical polynomial $F$ we associate two useful geometric objects: the Newton polytope $\Delta(F)$, defined as the convex hull of the set $\Omega$, and the tropical hypersurface $Z(F)$, defined as the corner locus of the graph of $F$, i.e., the set of points $x \in \mathbb{R}^{n}$ such that $F$ is not differentiable at $x$, or, equivalently, the set of points $x \in \mathbb{R}^{n}$ at which the maximum in the right-hand side of (1.1) is attained at least twice. A tropical hypersurface is a finite polyhedral object in $\mathbb{R}^{n}$ of pure dimension $n-1$, whose complement consists of open convex polyhedra (see [2, 3, 15]). It is the tropical analogue of the zero locus of a classical polynomial. We notice in addition that a tropical polynomial $F$ is differentiable (in the usual sense) outside $Z(F)$, and its partial derivatives are constant on each of the components of the complement of $Z(F)$.

The space of tropical polynomials in $n$ variables is denoted by " $\mathbb{R}\left[x_{1}, \ldots, x_{n}\right] " 1$ For $F_{1}, \ldots, F_{k} \in$ " $\mathbb{R}\left[x_{1}, \ldots, x_{n}\right]$ ", we define the tropical polynomial ideal $I\left(F_{1}, \ldots, F_{k}\right)$, generated by $F_{1}, \ldots, F_{k}$ in " $\mathbb{R}\left[x_{1}, \ldots, x_{n}\right]$ ", as the set of tropical polynomials $G \in$ " $\mathbb{R}\left[x_{1}, \ldots, x_{n}\right]$ " representable in the form

$$
G(x)=" \sum_{i \in J} h_{i}(x) F_{i}(x) "=\max _{i \in J}\left(h_{i}(x)+F_{i}(x)\right),
$$

where $J$ is a finite non-empty subset of $\{1,2, \ldots, k\}$, and $h_{i} \in$ " $\mathbb{R}\left[x_{1}, \ldots, x_{n}\right] ", i \in J$. The radical $\sqrt{I}$ of an ideal $I$ is the set of polynomials $G$ such that " $G$ " " $=m G \in I$ for some natural $m$. We ask the following question:

Given a tropical polynomial $F \in$ " $\mathbb{R}\left[x_{1}, \ldots, x_{n}\right] "$, under what conditions does $F$ belong to $\sqrt{I\left(F_{1}, \ldots, F_{k}\right)}$ ?

The answer, presented in the next section, is given in terms of the "zero loci" of the tropical polynomials $F, F_{1}, \ldots, F_{k}$, like in the classical case.

\section{A tropical polynomial Nullstellensatz}

Theorem 2.1. Let $F, F_{1}, \ldots, F_{k}, k \geq 1$, be tropical polynomials in $n$ variables. Then $F \in \sqrt{I\left(F_{1}, \ldots, F_{k}\right)}$ if and only if, for any connected component $D$ of $\mathbb{R}^{n} \backslash Z(F)$, there is $1 \leq i \leq k$ such that $D \cap Z\left(F_{i}\right)=\emptyset$, and, for each $j=1, \ldots, n$,

$$
\left.\frac{\partial F}{\partial x_{j}}\right|_{D}>0 \text { as far as }\left.\frac{\partial F_{i}}{\partial x_{j}}\right|_{D}>0 .
$$

Proof. 1. Necessity. The function $\left.m F\right|_{D}$ is linear. Hence it must coincide with one of the terms $\left.\left(h_{i}+F_{i}\right)\right|_{D}$ in the expression $\left.\max _{i}\left(h_{i}+F_{i}\right)\right|_{D}$, since otherwise the graph of the latter function would have a break inside $D$. Next, if $\left.m F\right|_{D}=\left.\left(h_{i}+F_{i}\right)\right|_{D}$, then both $\left.h_{i}\right|_{D}$ and $\left.F_{i}\right|_{D}$ must be linear in view of $Z\left(h_{i}+F_{i}\right) \supset Z\left(h_{i}\right) \cup Z\left(F_{i}\right)$. Thus, $D \cap Z\left(F_{i}\right)=\emptyset$. Observing that

$$
\left.m \frac{\partial F}{\partial x_{j}}\right|_{D}=\left.\frac{\partial h_{i}}{\partial x_{j}}\right|_{D}+\left.\frac{\partial F_{i}}{\partial x_{j}}\right|_{D} \geq\left.\frac{\partial F_{i}}{\partial x_{j}}\right|_{D},
$$

we obtain (2.1).

\footnotetext{
${ }^{1}$ The quotation marks mean that we supply this space with the tropical operations, maximum and summation. In turn, operations without quotations (including derivatives) have the usual meaning.
} 
2. Sufficiency. Distribute the connected components of $\mathbb{R}^{n} \backslash Z(F)$ into disjoint subsets $\Pi_{i}, i \in J$, where $J \subset\{1, \ldots, k\}$, such that, for any $i \in J$ and $D \in \Pi_{i}$, we have $Z\left(F_{i}\right) \cap D=\emptyset$ and relation (2.1).

Fix some $i \in J$. Condition (2.1) yields that there is $m_{1}$ such that, for any $m \geq m_{1}$, one has

$$
\left.m \frac{\partial F}{\partial x_{j}}\right|_{D} \geq\left.\frac{\partial F_{i}}{\partial x_{j}}\right|_{D}, \quad D \in \Pi_{i}, j=1, \ldots, n,
$$

and hence the gradients of the linear functions

$$
L_{D, m}: \mathbb{R}^{n} \rightarrow \mathbb{R},\left.\quad L_{D, m}\right|_{D}=\left.m F\right|_{D}-\left.F_{i}\right|_{D}, \quad D \in \Pi_{i}, m \geq m_{1},
$$

have non-negative integral coordinates.

We claim that there exists $m_{2}$ such that, for any $D \in \Pi_{i}$, in the complement of the closure of $D$, we have

$$
m F>L_{D, m}+F_{i} \quad \text { as far as } m \geq m_{1} .
$$

Indeed, write $F=L+\Phi, F_{i}=L^{\prime}+\Phi^{\prime}$, where $L, L^{\prime}$ are linear functions, and $\Phi, \Phi^{\prime}$ are convex piecewise linear functions, vanishing along $D$. Then $L_{D, m}=m L-L^{\prime}$, and thus, $m F-L_{D, m}-F_{i}=m \Phi-\Phi^{\prime}$. Since $\Phi>0$ and $\left|\partial \Phi / \partial x_{j}\right| \geq 1, j=1, \ldots, n$, outside $\bar{D}$, we obtain (2.3) when $m_{2}$ exceeds all the absolute values of the partial derivatives of $\Phi^{\prime}$.

Define $h_{i}=\max _{D \in \Pi_{i}} L_{D, m}$. This is a tropical polynomial as $m \geq m_{1}$, and due to (2.2), (2.3) it satisfies

$$
\left.\left(h_{i}+F_{i}\right)\right|_{D}=\left.m F\right|_{D},\left.\quad\left(h_{i}+F_{i}\right)\right|_{\mathbb{R}^{n} \backslash \bar{D}}<\left.F\right|_{\mathbb{R}^{n} \backslash \bar{D}}, \quad D \in \Pi_{i}, \quad m \geq m_{2} .
$$

That is, $G=" F^{m} "=m F$ satisfies (1.2) for all sufficiently large $m$.

Remark 2.2. From the above proof, one can extract an explicit upper bound to the minimal value of $m$ such that " $F m$ " $\in I\left(F_{1}, \ldots, F_{k}\right)$.

Example 2.3. In the case $k=1$, the criterion of Theorem 2.1 for $F \in \sqrt{I\left(F_{1}\right)}$ can be written as

- $Z(F) \supset Z\left(F_{1}\right)$, and

- for each $j=1, \ldots, n, \partial F / \partial x_{j}(x)>0$ as far as $\partial F_{1} / \partial x_{j}(x)>0, x \notin Z(F)$.

We shall comment on the first condition. One can assign integer positive weights to the $(n-1)$-cells of a tropical hypersurface so that it will satisfy an equilibrium condition (see 12 , section 2.1). Taking $m$ greater than the maximal ratio of the weights in $Z(F)$ and in $Z\left(F_{1}\right)$, we then subtract the weight of an $(n-1)$-cell $C$ of $Z\left(F_{1}\right)$ from the multiplied by $m$ weights of those $(n-1)$-cells of $Z(F)$, whose interior intersects with $C$. The equilibrium condition persists after the subtraction due to its linearity, and we again obtain a balanced complex supported at $Z(F)$. By [12, Proposition 2.4, it defines a convex piecewise linear function $h_{1}$, which provides the relation $m F=h_{1}+F_{1}$.

\section{Modifications}

3.1. A tropical Laurent polynomial Nullstellensatz. A tropical Laurent polynomial in $n$ variables is a function given by (1.1), where $\Omega$ is any non-empty finite subset of $\mathbb{Z}^{n}$. Denote the space of tropical Laurent polynomials by " $L\left[x_{1}, \ldots, x_{n}\right]$ ". Correspondingly we define the tropical Laurent polynomial ideal $I^{\text {lau }}\left(F_{1}, \ldots, F_{k}\right) \subset$ 
" $L\left[x_{1}, \ldots, x_{n}\right]$ ", generated by Laurent polynomials $F_{1}, \ldots, F_{k}$, as the set of tropical Laurent polynomials representable in the form (1.2) for some non-empty finite $J \subset\{1, \ldots, k\}$, and $h_{i} \in$ "L[x $\left[x_{1}, \ldots, x_{n}\right] ", i \in J$.

Theorem 3.1. Let $F, F_{1}, \ldots, F_{k}, k \geq 1$, be tropical Laurent polynomials in $n$ variables. Then $F \in \sqrt{I^{\mathrm{lau}}\left(F_{1}, \ldots, F_{k}\right)}$ if and only if, for any connected component $D$ of $\mathbb{R}^{n} \backslash Z(F)$, there is $1 \leq i \leq k$ such that $D \cap Z\left(F_{i}\right)=\emptyset$.

Proof. The necessity is established in the same way as in the proof of Theorem 2.1. The sufficiency follows from Theorem 2.1 if one "multiplies" $F, F_{1}, \ldots, F_{k}$ by suitable tropical monomials, turning $F, F_{1}, \ldots, F_{k}$ into tropical polynomials, and making all the partial derivatives of $F$ positive.

3.2. Restricted ideals and restricted Nullstellensatz. In the space " $\mathbb{R}\left[x_{1}, \ldots, x_{n}\right]$ " introduce the restricted ideal, generated by the tropical polynomials $F_{1}, \ldots, F_{k}$, as

$$
\begin{gathered}
I^{r}\left(F_{1}, \ldots, F_{k}\right)=\left\{G \in " \mathbb{R}\left[x_{1}, \ldots, x_{n}\right] ":\right. \\
\left.G=" \sum_{i=1}^{k} h_{i} F_{i} "=\max _{1 \leq i \leq k}\left(h_{i}+F_{i}\right), \quad h_{1}, \ldots, h_{k} \in " \mathbb{R}\left[x_{1}, \ldots, x_{n}\right] "\right\} .
\end{gathered}
$$

Notice that $I^{r}\left(F_{1}, \ldots, F_{k}\right) \subset I\left(F_{1}, \ldots, F_{k}\right)$. In general these ideals may differ, for example, $x \notin I^{r}(x, " x+1 ")$.

Theorem 3.2. Let $F, F_{1}, \ldots, F_{k}, k \geq 1$, be tropical polynomials in $n$ variables. Then $F \in \sqrt{I^{r}\left(F_{1}, \ldots, F_{k}\right)}$ if and only if the following conditions hold:

(i) for any connected component $D$ of $\mathbb{R}^{n} \backslash Z(F)$, there is $1 \leq i \leq k$ such that $D \cap Z\left(F_{i}\right)=\emptyset$ and (2.1) is fulfilled;

(ii) there is $m_{0}$ such that $m \Delta(F)$ contains a translate of each Newton polytope $\Delta\left(F_{1}\right), \ldots, \Delta\left(F_{k}\right)$ as far as $m \geq m_{0}$.

Remark 3.3. Notice that condition (ii) always holds when $\operatorname{dim} \Delta(F)=n$.

Proof. 1. Auxiliary statement. Let $G, H \in$ "R $\left[x_{1}, \ldots, x_{n}\right]$ ". We claim that

- if $G \geq H$, then $\Delta(G) \supset \Delta(H)$;

- if $\Delta(G) \supset \Delta(H)$, then there is $c \in \mathbb{R}$ such that $G \geq H+c$.

Represent $\Delta(G)$ as the intersection of finitely many halfspaces. Pick one of these halfspaces and apply an integral-affine automorphism $Q$ of $\mathbb{R}^{n}$, taking the halfspace to $x_{n} \geq 0$. Since $G \circ Q^{-1} \geq H \circ Q^{-1}$, the Newton polytope $\Delta\left(H \circ Q^{-1}\right)=Q(\Delta(H))$ cannot contain points with a negative $n$-th coordinate. Indeed, otherwise we would have that, for $x_{1}, \ldots, x_{n-1}=$ const, $x_{n} \rightarrow-\infty$, the function $G\left(Q^{-1}(x)\right)$ does not increase, whereas $H\left(Q^{-1}(x)\right)$ tends to $+\infty$. Running over all halfspaces forming $\Delta(G)$, we conclude that $\Delta(G) \supset \Delta(H)$.

For the second statement, write

$$
G(x)=\max _{\omega \in \Delta(G) \cap \mathbb{Z}^{n}}\left(\langle x, \omega\rangle+a_{\omega}\right), \quad H(x)=\max _{\omega \in \Delta(H) \cap \mathbb{Z}^{n}}\left(\langle x, \omega\rangle+b_{\omega}\right) .
$$

Then one can take

$$
c=\min _{\omega \in \Delta(H) \cap \mathbb{Z}^{n}}\left(a_{\omega}-b_{\omega}\right) .
$$

2. Necessity. We have to prove only (ii). Observing that $\Delta\left(h_{i}+F_{i}\right)$ is the convex hull of few translates of $\Delta\left(F_{i}\right)$, we derive (ii) from the above auxiliary statement. 
3. Sufficiency. A translate of $\Delta\left(F_{i}\right)$ is the Newton polytope of a tropical polynomial " $h_{i} F_{i}$ ", where $h_{i}$ is a tropical monomial (i.e., a linear function). Then, according to the auxiliary statement, $m F_{i} \geq\left(h_{i}+c_{i}\right)+F_{i}$ for sufficiently large $m$ and certain constant $c_{i}$. Then, using Theorem 2.1, we obtain

$$
m F=\max _{i \in J}\left(h_{i}+F_{i}\right)=\max \left\{\max _{i \in J}\left(h_{i}+F_{i}\right), \max _{i \notin J}\left(h_{i}+c_{i}+F_{i}\right)\right\} .
$$

3.3. Nullstellensatz for convex piecewise linear functions of finite type. A function given by (1.1), where $\Omega$ is a finite subset of $\mathbb{R}^{n}$, we call a convex piecewise linear function of finite type in $n$ variables. Denote the space of such functions by " $P L\left(x_{1}, \ldots, x_{n}\right)$ ". As in the polynomial case, we define tropical hypersurfaces $Z(F)$ in $\mathbb{R}^{n}$ and finitely generated ideals $I^{\text {fun }}\left(F_{1}, \ldots, F_{k}\right)$ in " $P L\left(x_{1}, \ldots, x_{n}\right)$ ", and obtain a corresponding Nullstellensatz:

Theorem 3.4. Let $F, F_{1}, \ldots, F_{k} \in$ "PL $\left(x_{1}, \ldots, x_{n}\right)$ ". Then $F \in \sqrt{I^{\text {fun }}\left(F_{1}, \ldots, F_{k}\right)}$ if and only if, for any connected component $D$ of $\mathbb{R}^{n} \backslash Z(F)$, there is $1 \leq i \leq k$ such that $D \cap Z\left(F_{i}\right)=\emptyset$.

The proof coincides with that of Theorem 3.1 .

3.4. Nullstellensatz for polynomials over an extended tropical semiring. In 6], the second author introduced an extension $\mathbb{T}$ of the tropical semiring $\mathbb{R}$. Namely, $\mathbb{T}$ is obtained by adding another copy of $\mathbb{R}$, which we denote by $\mathbb{R}^{\nu}$. That means $\mathbb{T}$ is the disjoint union of $\mathbb{R}$ and $\mathbb{R}^{\nu}$. Denoting elements of $\mathbb{R}^{\nu}$ by $a^{\nu} \in \mathbb{R}^{\nu}$, where $a \in \mathbb{R}$, we equip $\mathbb{T}$ with the following tropical operations:

$$
\begin{gathered}
" a+b "=\left\{\begin{array}{ll}
\max \{a, b\}, & \text { if } a \neq b, \\
a^{\nu}, & \text { if } a=b,
\end{array} \quad " a^{\nu}+b^{\nu} "=(\max \{a, b\})^{\nu},\right. \\
" a+b^{\nu} "= \begin{cases}a, & \text { if } a>b, \\
b^{\nu}, & \text { if } a \leq b,\end{cases} \\
" a b "=a+b, \quad " a^{\nu} b^{\nu} "=(a+b)^{\nu}, \quad " a b^{\nu} "=(a+b)^{\nu}
\end{gathered}
$$

for all $a, b \in \mathbb{R}$. There is a natural epimorphism of tropical semirings:

$$
\pi: \mathbb{T} \rightarrow \mathbb{R}, \quad \pi(a)=a, \pi\left(a^{\nu}\right)=a, \quad \text { for all } a \in \mathbb{R},
$$

which induces epimorphisms $\pi_{*}$ of the tropical polynomial and tropical Laurent polynomial rings. The above variants of Nullstellensatz can easily be translated to the case of base semiring $\mathbb{T}$. Here we present such a translation of Theorem 2.1.

For a polynomial $F=" \sum_{\omega \in \Omega} c_{\omega} x^{\omega} " \in$ " $\mathbb{T}\left[x_{1}, \ldots, x_{n}\right] "$, where $\Omega$ is a non-empty finite set of points with non-negative integral coordinates, we introduce a tropical hypersurface $Z(F):=Z\left(\pi_{*} F\right) \subset \mathbb{R}^{n}$. The restriction of $\pi_{*} F$ to a connected component $D$ of $\mathbb{R}^{n} \backslash Z(F)$ is a linear function $\langle\omega, x\rangle+\pi\left(c_{\omega}\right)$ for some $\omega=\omega(D) \in \Omega$. So we divide the set of the connected components of $\mathbb{R}^{n} \backslash Z(F)$ into two disjoint subsets $\Pi(F)$ and $\Pi^{\nu}(F)$, letting $D \in \Pi(F)$ or $D \in \Pi^{\nu}(F)$ according as $c_{\omega(D)} \in \mathbb{R}$ or $c_{\omega(D)} \in \mathbb{R}^{\nu}$.

Theorem 3.5. Let $F, F_{1}, \ldots, F_{k} \in$ "TT $\left[x_{1}, \ldots, x_{n}\right]$ ", $k \geq 1$. Then $F \in \sqrt{I\left(F_{1}, \ldots, F_{k}\right)}$ if and only if

(i) for any $D \in \Pi(F)$, there is $1 \leq i \leq k$ such that $D$ is contained in some component $D_{i} \in \Pi\left(F_{i}\right)$, and, for each $j=1, \ldots, n$, relation (2.1) holds true; 
(ii) for any $D \in \Pi^{\nu}(F)$, there is $1 \leq i \leq k$ such that $D \cap Z\left(F_{i}\right)=\emptyset$, and, for each $j=1, \ldots, n$, relation (2.1) holds true.

Proof. The necessity part is immediate in view of the proof of Theorem 2.1 and the fact that " $\mathbb{R} \mathbb{R}^{\nu} "=\mathbb{R}^{\nu}$. To prove the sufficiency, we construct coefficients $h_{i}$ of the expansion (1.2) to be in " $\mathbb{R}\left[x_{1}, \ldots, x_{n}\right]$ " $\subset$ " $\mathbb{T}\left[x_{1}, \ldots, x_{n}\right]$ " by the following modification of the procedure from the proof of Theorem 2.1

- if $D \in \Pi(F)$, and $1 \leq i \leq k$ satisfies condition (i), then we put $\left.h_{i}\right|_{D}=$ $\left.m F\right|_{D}-\left.F_{i}\right|_{x} D$

- if $D \in \Pi^{\nu}(F)$, and $1 \leq i \leq k$ satisfies condition (ii), then we put $\left.h_{i}\right|_{D}=$ $\left.m \cdot \pi_{*} F\right|_{D}-\left.\pi_{*} F_{i}\right|_{D}$.

Choosing a sufficiently large $m$, we complete the proof as that of Theorem 2.1

Corollary 3.6. Let $F, F_{1}, \ldots, F_{k} \in$ "T $\left[x_{1}, \ldots, x_{n}\right] ", k \geq 1$, and $I\left(F_{1}, \ldots, F_{k}\right) \supset$ " $\mathbb{R}^{\nu}\left[x_{1}, \ldots, x_{n}\right]$ ". Then $F \in \sqrt{I\left(F_{1}, \ldots, F_{k}\right)}$ if and only if condition (i) of Theorem 3.5 holds true.

Indeed, the relation $I\left(F_{1}, \ldots, F_{k}\right) \supset$ " $\mathbb{R}^{\nu}\left[x_{1}, \ldots, x_{n}\right]$ " means that the set of generators $\left\{F_{1}, \ldots, F_{k}\right\}$ contains a constant polynomial $F_{i}=a^{\nu}, a \in \mathbb{R}$, and thus, condition (ii) always holds.

\section{ACKNOWLEDGMENTS}

We thank an unknown referee for valuable remarks, which allowed us to correct defects and improve the readability of the text.

\section{REFERENCES}

1. Amoebas and tropical geometry. Preprint available at http://www.aimath.org/WWN/ amoebas.

2. M. Einsiedler, M. Kapranov, and D. Lind, Non-Archimedean amoebas and tropical varieties. Preprint at arXiv:math.AG/0408311.

3. I. Itenberg, Amibes des variétés algébriques et denombrement de courbes [d'après G. Mikhalkin]. Séminaire N. Bourbaki 921, vol. 2002-03, Juin 2003.

4. I. Itenberg, V. Kharlamov, and E. Shustin, Welschinger invariant and enumeration of real rational curves. International Math. Research Notices 49 (2003), 2639-2653. MR2012521 (2004h:14065)

5. I. Itenberg, V. Kharlamov, and E. Shustin, Logarithmic equivalence of Welschinger and Gromov-Witten invariants. Russian Math. Surveys 59 (2004), no. 6, 1093-1116. MR 2138469 (2006b:14101)

6. Z. Izhakian, Tropical Arithmetic and Algebra of Tropical Matrices. Preprint at arXiv:math.AG/0505458.

7. V. N. Kolokoltsov and V. P. Maslov, Idempotent Analysis and Applications. Kluwer Acad. Publ., Dordrecht, The Netherlands, 1997.

8. M. Kontsevich and Y. Soibelman, Homological mirror symmetry and torus fibrations. Symplectic geometry and mirror symmetry (Seoul, 2000), World Sci. Publishing, River Edge, NJ, 2001, pp. 203-263. MR.1882331 (2003c:32025)

9. M. Kontsevich, M. and Yu. Tschinkel, Nonarchimedean Kähler geometry. Preprint, 2002.

10. G. Mikhalkin, Counting curves via the lattice paths in polygons. C. R. Acad. Sci. Paris, Sér. I, 336 (2003), no. 8, 629-634. MR.1988122(2004d:14077)

11. G. Mikhalkin, Amoebas of algebraic varieties and tropical geometry. Different faces of geometry/Donaldson, S. (ed.) et al. Kluwer, NY, 2004, pp. 257-300. MR2102998(2005m:14110)

12. G. Mikhalkin, Decomposition into pairs-of-pants for complex algebraic hypersurfaces. Topology 43 (2004), 1035-1065. MR2079993 (2005i:14055) 
13. G. Mikhalkin, Enumerative tropical algebraic geometry in $\mathbb{R}^{2}$. J. Amer. Math. Soc. 18 (2005), 313-377. MR2137980 (2006b:14097)

14. E. Shustin, A tropical approach to enumerative geometry. Algebra i Analiz 17 (2005), no. 2, 170-214 (English translation: St. Petersburg Math. J. 17 (2006), 343-375). MR.2159589 (2006i:14058)

15. D. Speyer and B. Sturmfels, The tropical Grassmannian. Adv. Geom. 4 (2004), 389-411. MR2071813 (2005d:14089)

16. D. Speyer and B. Sturmfels, Tropical Mathematics. Preprint arXiv:math.CO/0408099.

17. O. Viro, Dequantization of Real Algebraic Geometry on a Logarithmic Paper. Proceedings of the 3rd European Congress of Mathematicians, Birkhäuser, Progress in Math, 201, (2001), 135-146. MR1905317 (2003f:14067)

School of Mathematical Sciences, Tel Aviv University, Ramat Aviv, 69978 Tel Aviv, ISRAEL

E-mail address: shustin@post.tau.ac.il

School of Computer Sciences, Tel Aviv University, Ramat Aviv, 69978 Tel Aviv, ISRAEL

E-mail address: zzur@post.tau.ac.il 